\title{
Shunting Inhibition Does Not Have a Divisive Effect on Firing Rates*
}

\author{
Gary R. Holt \\ Christof Koch \\ Computation and Neural Systems Program, California Institute of Technology, \\ Pasadena, CA 91125, U.S.A.
}

Shunting inhibition, a conductance increase with a reversal potential close to the resting potential of the cell, has been shown to have a divisive effect on subthreshold excitatory postsynaptic potential amplitudes. It has therefore been assumed to have the same divisive effect on firing rates. We show that shunting inhibition actually has a subtractive effect on the firing rate in most circumstances. Averaged over several interspike intervals, the spiking mechanism effectively clamps the somatic membrane potential to a value significantly above the resting potential, so that the current through the shunting conductance is approximately independent of the firing rate. This leads to a subtractive rather than a divisive effect. In addition, at distal synapses, shunting inhibition will also have an approximately subtractive effect if the excitatory conductance is not small compared to the inhibitory conductance. Therefore regulating a cell's passive membrane conductance-for instance, via massive feedback-is not an adequate mechanism for normalizing or scaling its output.

\section{Introduction}

Many neuronal models treat the output of a neuron as an analog value coded by the firing rate of a neuron. Often the analog value is thought of as what the somatic voltage would be if spikes were pharmacologically disabled (sometimes called a generator potential). This has led to a class of simplified single-compartment models where the steady-state above-threshold membrane potential is computed as

$$
V=\frac{I_{\text {syn }}}{G}
$$

\footnotetext{
* It has come to our attention that a previous study also pointed out that shunting synapses close to the spike mechanism have a subtractive effect (F. Gabbiani, J. Midtgaard, and T. Knöppel, Synaptic Integration in a Model of Cerebellar Granule Cells, J. Neurophysiol., 72: 999-1009, 1994).
} 


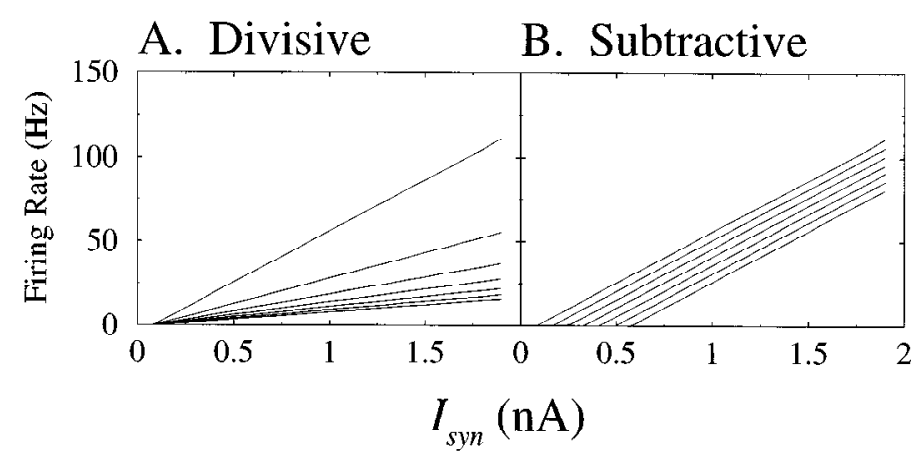

Figure 1: A comparison of divisive and subtractive inhibition. (A) Divisive inhibition changes the slope of the input-output relationship. In this case, $f=g(V)$ was a linear function of $V$ and $G$ was varied from 10 to $70 \mathrm{nS}$ in equal steps. (B) Subtractive inhibition shifts the curves by subtracting a current. Here $I_{\text {inh }}$ varies from 0.08 to $0.058 \mathrm{nA}$ in equal steps.

where $I_{s y n}$ is the synaptic current and $G$ is the input conductance. A firing rate is then computed directly from this above-threshold membrane potential:

$$
f=g(V)
$$

where $g$ is some monotonic function-for example, $f_{\text {out }} \propto V^{2}$ in Carandini and Heeger (1994) or $f_{\text {out }}=\tanh (V)$ in Hopfield (1984).

Varying $G$ - for instance, via activation of inhibitory input with a reversal potential close or equal to the cell's resting potential (also known as silent or shunting inhibition)—will directly affect the generator potential $V$ in a divisive manner. A recent and quite popular model (Carandini \& Heeger, 1994; Nelson, 1994) has suggested that changing $G$ by shunting inhibition would be a useful way to control the gain of a cell; when the inhibitory input rate increases, the slope of the input-output relationship decreases (see Figure 1A), but the threshold does not change much.

On the other hand, inhibition that is not of the shunting variety should have a subtractive effect on the input-output relationship. If the reversal potential of the inhibition is far from the spiking threshold, then the inhibitory synapse will act more like a current source; the cell's conductance is not changed much, but a hyperpolarizing current is injected. This current simply shifts the input-output relationship by changing $I_{s y n}$ to $I_{s y n}-I_{\text {inh }}$ (see Figure 1B) where $I_{\text {inh }}$ is the inhibitory current.

Simplified models based on a generator potential ignore the effect of the spiking mechanism and assume that the behavior of the neuron above threshold is adequately described by the subthreshold equations. We show that because of the spiking mechanism, changing the membrane leak con- 
ductance by shunting inhibition does not have a divisive effect on firing rate, casting doubt on the hypothesis that such a mechanism serves to normalize a cell's response. A similar conclusion has been reached independently for certain classes of neuron models (Payne \& Nelson, 1996).

\section{Methods}

Compartmental simulations were done using the model described by Bernander, Douglas, Martin, and Koch (1991); Bernander, Koch, and Douglas (1994); Bernander (1993); and Koch, Bernander, and Douglas (1995). The geometry for the compartmental models was derived from a large layer $\mathrm{V}$ pyramidal cell and a much smaller layer IV spiny stellate cell stained during in vivo experiments in cat (Douglas, Martin, \& Whitteridge, 1991) and reconstructed. Geometries of both cells are shown as insets in Figure 2. Each model has the same eight active conductances at the soma, including an A current and adaptation currents (see Koch et al., 1995, for details). The somatic conductance values were different for each cell, but the same conductance per unit area was used for each type of channel. Dendrites were passive. Simulations were performed with the program Neuron (Hines, 1989, 1993).

To study the effect of $\mathrm{GABA}_{\mathrm{A}}$ synapses and shunting inhibition, we did not explicitly model each synapse; we set the membrane leak conductance and reversal potential at each location in the dendritic tree to be the timeaveraged values expected from excitatory and inhibitory synaptic bombardment at presynaptic input firing rates $f_{E}$ and $f_{I}$ (as described in Bernander et al., 1991). To compute the time-averaged values, synapses were treated as alpha functions with a given time constant and maximum conductance $\left(g(t)=g_{\max } t e^{-t / \tau} e^{-1} / \tau\right)$. The area density of synapses at a given location on a dendrite was a function of the length of dendrite that separated the area from the soma (see Table 1). Two different sets of densities ("near" and "far") were used, depending on whether inhibitory synapses were near the soma or far from the soma. The "near" configuration is identical to the distribution used by Bernander et al. (1991) and reflects the anatomical observation that inhibitory synapses are mostly located near the soma in cortical pyramidal cells. The "far" configuration is not intended to be anatomically realistic. For simplicity, we used the same number of synapses for both the spiny stellate cell and the layer $\mathrm{V}$ pyramidal cell models.

Our integrate-and-fire model is described by

$$
C \frac{d V}{d t}=-V g_{\text {leak }}+I_{\text {syn }} \quad \text { for } V<V_{t h}
$$

where $g_{\text {leak }}$ is the input conductance, $C$ is the capacitance, and $I_{\text {syn }}$ is the synaptic input. When the voltage $V$ exceeds a threshold $V_{t h}$, the cell emits a spike and resets its voltage back to 0 . We used $C=1 \mathrm{nF}$, $g_{\text {leak }}=16 \mathrm{nS}$, $V_{\text {th }}=16.4 \mathrm{mV}$, which matches the adapted current-discharge curve of the 
Table 1: Parameters of Synapses in the Compartmental Models

\begin{tabular}{llllllc}
\hline Type & Reversal & Number & $g_{\max }$ & $\tau$ & \multicolumn{2}{c}{ Area Density } \\
\cline { 5 - 6 } & Potential & & & & Near & Far \\
\hline AMPA & $0 \mathrm{mV}$ & 4000 & $1 \mathrm{nS}$ & $0.5 \mathrm{~ms}$ & $1+\tanh \left(\frac{x-40}{22.73}\right)$ & $1+\tanh \left(\frac{x-100}{22.73}\right)$ \\
GABA $_{A}$ & $-70 \mathrm{mV}$ & 500 & $1 \mathrm{nS}$ & $5 \mathrm{~ms}$ & $e^{-x / 50}$ & $1+\tanh \left(\frac{x-100}{22.73}\right)$ \\
GABA $_{B}$ & $-95 \mathrm{mV}$ & 500 & $0.1 \mathrm{nS}$ & $40 \mathrm{~ms}$ & & $x e^{-x / 50}$
\end{tabular}

Note: $x$ is the length of dendrite in $\mu \mathrm{m}$ that separates this compartment from the soma. The "near" area density was used for Figure 2; the "far" area density was used for Figure 4. The normalization for the area density is not included in the expression because it depends on the geometry; different neurons have different fractions of their membrane at a given distance from the soma.

layer V pyramidal cell model quite well (not shown). Results from this article are not changed if a refractory period or an adaptation conductance is added to the integrate-and-fire model.

\section{Results}

We first describe our results and reasoning for an "anatomically correct" distribution of synaptic inhibition in relative close proximity to the cell body. Since we fail to observe silent inhibition act divisively but would like to know under which circumstances it can act so, we next investigate the anatomically less realistic situation of more distal inhibition.

3.1 Proximal Inhibition. Changing $g_{\text {leak }}$ does not change the slope of the current-discharge curve for integrate-and-fire cells (see Figure 2A); it primarily shifts the curves. It therefore has a subtractive rather than a divisive effect.

The compartmental models' behavior is very similar to that of the integrate-and-fire unit. For two different geometries (a layer $\mathrm{V}$ pyramid and a layer IV spiny stellate cell), we computed the fully adapted firing rate as a function of the excitatory synaptic input rate for various different rates of inhibitory input to synapses with $\mathrm{GABA}_{\mathrm{A}}$ receptors (see Figure $2 \mathrm{~B}$ ). The slope of the input-output relationship does not change when the $\mathrm{GABA}_{\mathrm{A}}$ input amplitude is changed; the entire curve shifts. The same effect can be observed when considering the current-discharge curves (not shown).

This effect is most easily understood in the integrate-and-fire model. In the absence of any spiking threshold, $V$ would rise until $V=I_{\text {syn }} / g_{\text {leak }}$ (see Figure 3). Under these conditions, the steady-state leak current is propor- 

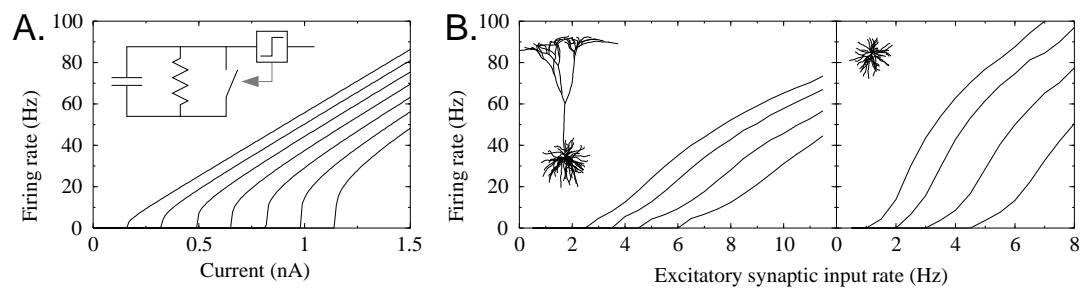

Figure 2: Changing $g_{\text {leak }}$ has a subtractive rather than a divisive effect on firing rates. (A) Current-discharge curves for the integrate-and-fire model, with gleak varying from 10 to $70 \mathrm{nS}$ (from left to right) in steps of $10 \mathrm{nS}$. (B) Fully adapted firing rates of the two cells as a function of excitatory input rate for different inhibitory input rates. From left to right, the curves correspond to a $\mathrm{GABA}_{\mathrm{A}}$ inhibitory rate of $0.5,2,4$, and $6 \mathrm{~Hz}$. In all of these cases, the curve shifts rather than changes slope. In this case, inhibitory synapses were near the soma ("near" configuration in Table 1), as found in cortical cells.

tional to the input current. However, if there is a spiking threshold, $V$ never rises above $V_{t h}$. Therefore, no matter how large the input current is, the leak current can never be larger than $V_{\text {th }} g_{\text {leak }}$. We can replace the leak conductance by a current whose value is equal to the time-average value of the current through the leak conductance $\left(\left\langle I_{\text {leak }}\right\rangle=g_{\text {leak }}\langle V\rangle\right)$, and simplify the leaky integrate-and-fire unit to a perfect integrator. (Now, however, $\left\langle I_{\text {leak }}\right\rangle$ will be a function of $I_{s y n}$.) If the current is suprathreshold, the cell will still fire at exactly the same rate because the same charge $\int_{0}^{T}\left(I_{\text {syn }}-I_{\text {leak }}\right) d t$ is deposited on the capacitor during one interspike interval $T$, although for the leaky integrator the deposition rate is not constant.

For constant just suprathreshold inputs, $\langle V\rangle$ will be close to $V_{\text {th }}$, and $\left\langle I_{\text {leak }}\right\rangle$ will be large. For larger synaptic input currents, the time-averaged membrane potential becomes less and less (since $V$ has to charge up from the reset point), and therefore the time-averaged leak current decreases for increasing inputs (compare Figures 3A and 3B). It can be shown that

$\left\langle I_{\text {leak }}\right\rangle= \begin{cases}I_{\text {syn }} & \text { if } I_{\text {syn }}<V_{\text {th }} g_{\text {leak }} \\ V_{\text {th }} g_{\text {leak }}\left(\frac{I_{\text {syn }}}{V_{\text {th }} g_{\text {leak }}}+\frac{1}{\log \left(1-V_{\text {th }} g_{\text {leak }} / I_{\text {syn }}\right)}\right) & \text { otherwise. }\end{cases}$

For large $I_{\text {syn }}$, and even for quite moderate levels of $I_{\text {syn }}$ just above $V_{\text {th }} g_{\text {leak }}$, the lower expression is approximately equal to $g_{\text {leak }} V_{t h} / 2$, independent of $I_{\text {syn }}$ (see Figure 3C). Therefore, it is a good approximation to replace the leak conductance by a constant offset current. The current-discharge curve for 
the resulting perfect integrate-and-fire neuron is simply ${ }^{1}$

$$
f(I)=\frac{I_{\text {syn }}-\left\langle I_{\text {leak }}\right\rangle}{C V_{\text {th }}}=\frac{I_{\text {syn }}}{C V_{\text {th }}}-\frac{g_{\text {leak }}}{2 C} .
$$

Shunting inhibition (varying $g_{\text {leak }}$ ) above threshold acts as a constant, hyperpolarizing current source, quite distinct from its subthreshold behavior.

A similar mechanism explains the result for the compartmental models. Here, the voltage rises above the firing threshold, but the spiking mechanism acts as a kind of voltage clamp on a long time scale (Koch et al., 1995) so that the time-averaged voltage remains approximately constant. Furthermore, spiking conductances are so large that during a spike, any proximal synaptic conductances will be ignored.

3.2 Distal Inhibition. Distal synapses are not so tightly coupled electrically to the soma, so one might expect that distal $\mathrm{GABA}_{\mathrm{A}}$ inhibition might act divisivly. Since the spiking mechanism can be thought of as a kind of voltage clamp (Koch et al., 1995), one can study the neuron's response by examining the current into the soma when it is clamped at the time-averaged voltage $V_{S}$ (Abbott, 1991). For analysis, we simplify the dendritic tree into a single finite cable that has an excitatory synapse (conductance $g_{E}$, reversal potential $\left.E_{E}\right)$ and an inhibitory synapse $\left(g_{I}\right.$ and $\left.E_{I}\right)$ located at the other end. The cable has length $l$, radius $r$, specific membrane conductance $G_{\text {leak }}$, intracellular resistivity $R_{i}$, and a length constant $\lambda=\sqrt{r /\left(2 R_{i} G_{\text {leak }}\right)}$. Using the cable equation, one can show that at steady state, the current flowing into the soma from the cable is

$$
\begin{aligned}
I_{\text {soma }}= & -g_{\infty} V_{s} \\
& +2 g_{\infty} e^{-L} \frac{g_{E}\left(E_{E}-V_{s} e^{-L}\right)+g_{I}\left(E_{I}-V_{s} e^{-L}\right)+g_{\infty} V_{s} e^{-L}}{\left(g_{E}+g_{I}\right)\left(1-e^{-2 L}\right)+g_{\infty}\left(1+e^{-2 L}\right)},
\end{aligned}
$$

where $L=l / \lambda$ is the electrotonic length of the cable and $g_{\infty}=\pi r^{3 / 2}$ $\sqrt{2 G_{\text {leak }} / R_{i}}$ is the input conductance of the cylinder but with infinite length. (In this equation, all voltages are relative to the leak reversal potential, not to ground.) Despite the simplification involved in equation 3.3, it qualitatively describes the response of the compartmental model.

First, in the absence of any cable $(L=0)$, this equation becomes linear in both $g_{E}$ and $g_{I}$, and inhibition acts to subtract a constant amount from $I_{\text {soma }}$, as we have shown above.

\footnotetext{
${ }^{1}$ This expression can also be derived from the Laurent expansion of the current discharge curve for a leaky integrator, $f\left(I_{\text {syn }}\right)=-g_{\text {leak }} / C \log \left(1-V_{\text {th }} g_{\text {leak }} / I_{\text {syn }}\right)$, in terms of $1 / I_{\text {syn }}$ around $I_{\text {syn }}=\infty$ (Stein, 1967).
} 


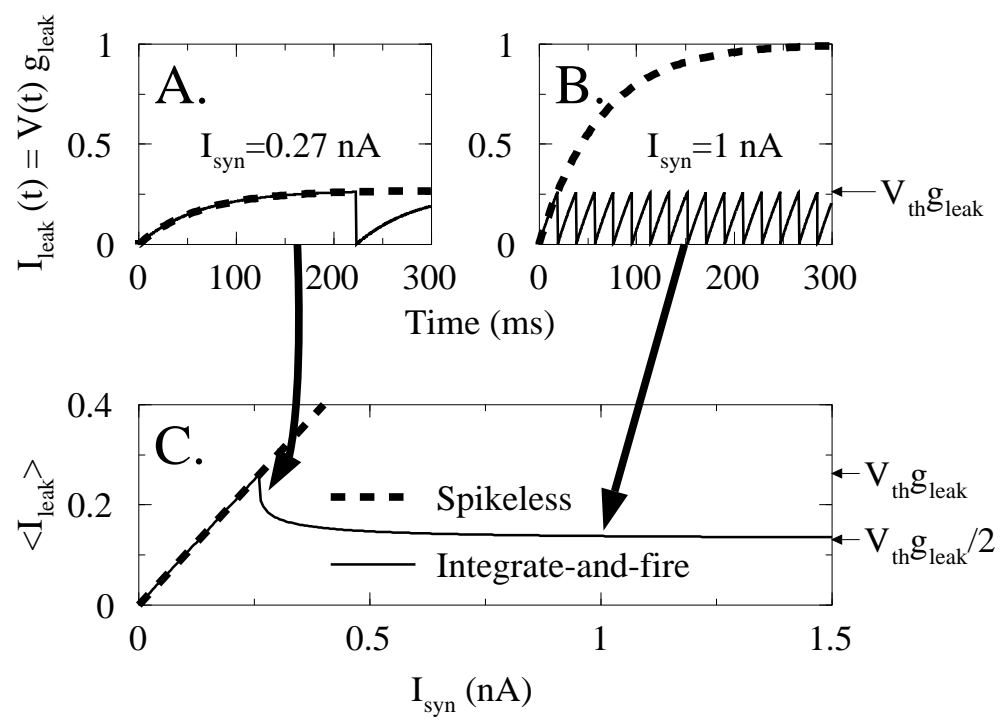

Figure 3: Why shunting inhibition has a subtractive rather than a divisive effect on an integrate-and-fire unit. (A) The time-dependent current across the leak conductance $I_{\text {leak }}$ (in $\mathrm{nA}$ ) in response to a constant $0.5 \mathrm{nA}$ current into a leaky integrate-and-fire unit with (solid line) and without (dashed line) a voltage threshold, $V_{t h}$. The sharp drops in $I_{\text {leak }}$ occur when the cell fires, since the voltage is reset. (B) Same for a $1 \mathrm{nA}$ current. Note that $I_{\text {leak }}$ with a voltage threshold has a maximum value well below $I_{\text {leak }}$ without a voltage threshold. (C) Timeaveraged leak current $\left\langle I_{\text {leak }}\right\rangle$ in $\mathrm{nA}$ as a function of input current, computed from the analytic expression. Below threshold, the spikeless model and the integrateand-fire models have the same $I_{\text {leak }}$, but above threshold $\left\langle I_{\text {leak }}\right\rangle$ drops for the integrate-and-fire model because of the voltage threshold. For $I_{\text {syn }}$ just greater than threshold, the cell spends most of its time with $V \approx V_{\text {th }}$, so $\left\langle I_{\text {leak }}\right\rangle$ is high (panel A; at threshold, $I_{\text {leak }}=V_{\text {th }} g_{\text {leak }}$ ). For high $I_{\text {syn }}$, the voltage increases approximately linearly with time, so $V$ has a sawtooth waveform as shown in panel B. This means that $\left\langle I_{\text {leak }}\right\rangle=\left(\max I_{\text {leak }}\right) / 2=V_{\text {th }} g_{\text {leak }} / 2$.

When $L \neq 0$, some divisive effect is expected since $g_{I}$ appears in the denominator. However, a subtractive effect will persist due to the term containing $g_{I}$ in the numerator. The reversal potential of $\mathrm{GABA}_{\mathrm{A}}$ synapses (increasing a membrane conductance to chloride ions) is in the neighborhood of $-70 \mathrm{mV}$ relative to ground, while the time-averaged voltage when the model neuron is spiking is around $-50 \mathrm{mV}$ (Koch et al., 1995). When a cell is spiking, therefore, a nonzero driving potential exists for $\mathrm{GABA}_{\mathrm{A}}$ inhibition. In the pyramidal cell model, the subtractive effect turns out to be much more prominent than the divisive effect even for quite distant inhibi- 


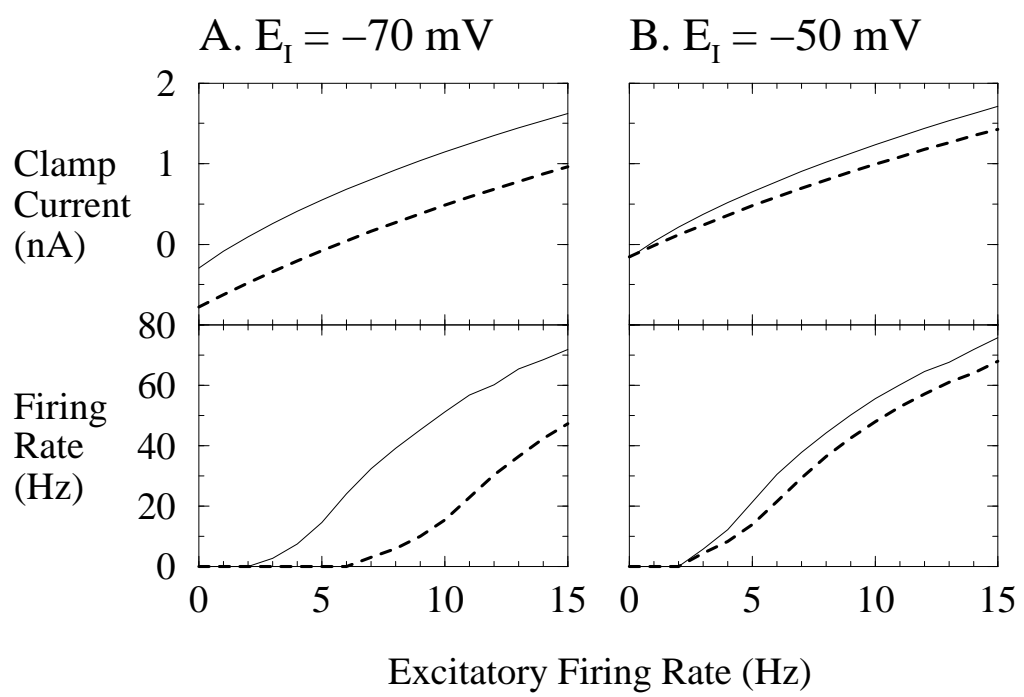

Figure 4: The effect of more distant inhibition on firing rates for the layer $\mathrm{V}$ pyramidal cell. (A) The effect on the input-output relationship when inhibitory synapses are more distant from the soma ("far" configuration in Table 1). Inhibitory rates are $0.5 \mathrm{~Hz}$ (solid) and $8 \mathrm{~Hz}$ (dashed). In these simulations, $E_{I}$, the $\mathrm{GABA}_{\mathrm{A}}$ reversal potential, had its usual value of $-70 \mathrm{mV}$. Top: The voltage clamp current when the soma is clamped to $-50 \mathrm{mV}$, close to the spiking threshold of the cell. Bottom: The adapted firing rate when the soma is not clamped. Very little divisive effect is visible on the firing rate; there is a slope change for firing rates less than $20 \mathrm{~Hz}$, but this is too small to have a significant effect. (B) Same as A, except that $E_{I}$ and the reversal potential of all leak conductances were changed to $-50 \mathrm{mV}$ so there is no driving force behind the $\mathrm{GABA}_{\mathrm{A}}$ synapses or the membrane passive conductance. A clear change in slope for low firing rates is evident. However, even for this rather unphysiological parameter manipulation, subtraction prevails at moderate and high input rates.

tion (see Figure 4A). Both the inhibitory and excitatory synapses have been moved to more than $100 \mu \mathrm{m}$ (which is more than $1 \lambda$ ) away from the soma. To a good approximation, inhibition still subtracts a constant from both the current delivered to the soma and the firing rate of the cell.

Equation 3.3 predicts that if the term containing $g_{I}$ is removed from the numerator, then a divisive effect might be visible. When we changed the reversal potential $E_{I}$ of the $\mathrm{GABA}_{\mathrm{A}}$ synapses and the leak reversal potential $E_{\text {leak }}$ to $-50 \mathrm{mV}$, we found that there is indeed an observable change in slope at a low firing rate (see Figure 4B). For higher firing rates, however, inhibition still acts approximately subtractively. We demonstrate this in the extreme case of moving both $E_{I}$ and the reversal potential associated with the leak 


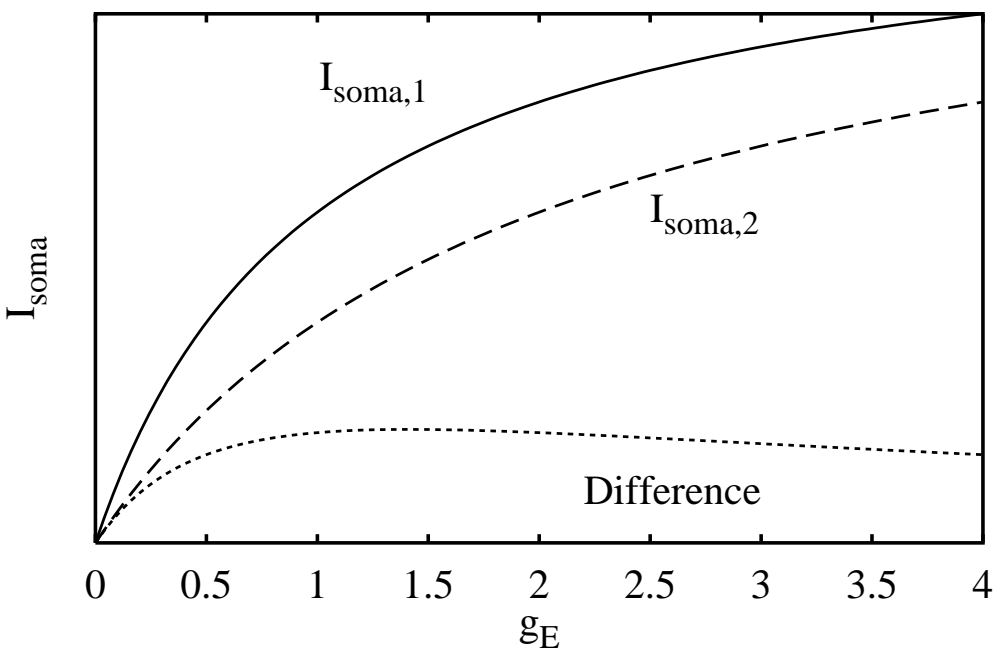

Figure 5: When $g_{E}$ is not small compared to $g_{I}$, then inhibition acts more subtractively than divisively even when the IPSP reversal potential is equal to the somatic voltage. The two upper curves are $I_{s o m a}$ as a function of $g_{E}$ for two different values of $g_{I}$ such that $g_{I}+B$ changes by a factor of two in equation 3.4. The lower curve is the difference between those two curves. For $g_{E}>g_{I}+B$, it is approximately constant over a large range. Units of $g_{E}$ are such that $g_{I}+B=1$ for the smaller value of $g_{I}$.

conductance to $-50 \mathrm{mV}$ (also the value to which the somatic terminal of the cable is clamped). Under these conditions, equation 3.3 simplifies to

$$
I_{\text {Soma }}\left(g_{E}, g_{I}\right)=\text { constant }+A \frac{g_{E}}{g_{E}+g_{I}+B}
$$

where $A$ and $B$ are independent of $g_{E}$ and $g_{I}$. Clearly if $g_{E}$ is small, changing $g_{I}$ simply changes the slope. When $g_{E}$ is not small, then it turns out that changing $g_{I}$ has an effect that is more subtractive than divisive (see Figure 5).

Since in the "far" model both kinds of synapses have the same distribution, their firing rates are proportional to the conductances. With the synaptic parameters we have used (see Table 1 ), $g_{E} / g_{I}=0.8 f_{E} / f_{I}$; therefore, we expect to see a subtractive effect when $g_{E}>6 \mathrm{~Hz}$, and this is approximately true (see Figure 4B).

\section{Discussion}

Divisive normalization of firing rates has become a popular idea in the visual cortex (Albrecht \& Geisler, 1991; Heeger, 1992; Heeger, Simoncelli, \& 
Movshon, 1996). It has been suggested that this is accomplished through shunting inhibitory synapses activated by cortical feedback (Carandini \& Heeger, 1994; for a similar suggestion in the electric fish, see Nelson, 1994). Most discussions of shunting inhibition have assumed that the voltage at the location of the shunt is not constrained and may rise as high as necessary (e.g., Blomfield, 1974; Torre \& Poggio, 1978; Koch, Poggio, \& Torre, 1982, 1983). However, when the shunt is located close to the soma, the voltage at the site of the shunt cannot rise above the spike threshold. Therefore, the current that can flow into the cell through the shunting synapse is limited and at moderate rates becomes a constant offset (see Figure 3C). Under these circumstances, shunting inhibition implements a linear subtractive operation.

Even if the conductance change is not located close to the soma, it may not have a divisive effect (see Figure 4A). First, when the cell is spiking, shunting inhibition is not "silent"; there is a significant driving force behind GABA inhibition, since the somatic voltage is clamped to approximately $-50 \mathrm{mV}$ by the spiking mechanism, and the reversal potential for $\mathrm{GABA}_{\mathrm{A}}$ inhibition is in the neighborhood of $-70 \mathrm{mV}$. Second, even if the reversal potential for $\mathrm{GABA}_{\mathrm{A}}$ and the leak reversal potential are set to $-50 \mathrm{mV}$, inhibition acts divisively only if the excitatory synaptic conductance is small compared to the inhibitory conductance (see Figures $4 \mathrm{~B}$ and 5 ). Large excitatory conductances are expected when the cell receives significant input (Bernander et al., 1991; Rapp, Yarom, \& Segev, 1992) so the subtractive effect of large conductances is relevant physiologically.

Current-discharge curves are affected as predicted by the simple integrate-and-fire model in response to inhibitory postsynaptic potential and GABA iontophoresis in motoneurons in vivo (Granit, Kernell, \& Lamarre, 1966; Kernell, 1969) and cortical cells in vitro (Connors, Malenka, \& Silva, 1988; Berman, Douglas, \& Martin, 1992). The input-output curves for different amounts of inhibition do not diverge for larger inputs, as would be required for a divisive effect; in fact, they converge at high rates because of the refractory period (Douglas \& Martin, 1990). In recordings from Limulus eccentric cells, current-discharge curves show both a slope change and a shift (Fuortes, 1959) because the site of current injection is distant from the site of spike generation. ${ }^{2}$ Rose (1977) showed that iontophoresis of $\mathrm{GABA}_{\mathrm{A}}$ onto an in vivo cortical network appeared to act divisively. Because shunting inhibition has a subtractive effect on single cells, this could possibly be caused by a network effect (Douglas, Koch, Mahowald, Martin, \& Suarez, 1995).

For synapses close to the spike-generating mechanism, as well as for the integrate-and-fire unit, the subtractive effect of conductance changes does

\footnotetext{
${ }^{2}$ In this case, only the current-discharge curve was measured; the considerations in Figures 4 and 5 are not relevant.
} 
not depend on the reversal potential of the conductance. Changing the reversal potential is equivalent to adding a constant current source in parallel with the conductance, and in a single compartment model this will obviously merely shift the current-discharge curve. Therefore, like inhibitory input, proximal excitatory input does not change the gain of other superimposed excitatory input.

Our analysis assumes that synaptic inputs change on a time scale slower than an interspike interval. High temporal frequencies may be present in synaptic input currents for irregularly spiking neurons. Furthermore, our analysis assumes passive dendrites; active dendritic conductances complicate the interaction of synaptic excitation and inhibition.

Although we cannot rule out that under some parameter combinations shunting inhibition could act divisively on the firing rates, we have not found such a range for physiological conditions. In combination with our integrate-and-fire and single cable models, we believe that a different mechanism is necessary to account for divisive normalization.

\section{Acknowledgments and Notes}

We thank the referees for helpful critical comments on this article. This research was supported by the NIMH and the Sloan Center for Theoretical Neuroscience.

The compartmental models and associated programs are available from: ftp:/ / ftp.klab.caltech.edu/pub/holt/holt_and_koch_1997_normalization. tar.gz.

\section{References}

Abbott, L. F. (1991). Realistic synaptic inputs for model neural networks. Network 2:245-258.

Albrecht, D. G., \& Geisler, W. S. (1991). Motion selectivity and the contrastresponse function of simple cells in the visual cortex. Vis. Neurosci. 7:531-546.

Berman, N. J., Douglas, R. J., \& Martin, K. A. C. (1992). GABA-mediated inhibition in the neural networks of visual cortex. Prog. Brain Res. 90:443-476.

Bernander, Ö. (1993). Synaptic integration and its control in neocortical pyramidal cells. Unpublished doctoral dissertation, California Institute of Technology, Pasadena.

Bernander, Ö., Douglas, R., Martin, K., \& Koch, C. (1991). Synaptic background activity determines spatio-temporal integration in single pyramidal cells. Proc. Natl. Acad. Sci. USA 88:1569-1573.

Bernander, Ö., Koch, C., \& Douglas, R. J. (1994). Amplification and linearization of distal synaptic input to cortical pyramidal cells. J. Neurophysiol. 72:27432753.

Blomfield, S. (1974). Arithmetical operations performed by nerve cells. Brain Res. 69:114-124. 
Carandini, M., \& Heeger, D. J. (1994). Summation and division by neurons in primate visual cortex. Science 264:1333-1335.

Connors, B. W., Malenka, R. C., \& Silva, L. R. (1988). Two inhibitory postsynaptic potentials, and $\mathrm{GABA}_{A}$ and $\mathrm{GABA}_{B}$ receptor-mediated responses in neocortex of rat and cat. J. Physiol. 406:443-468.

Douglas, R. J., Koch, C., Mahowald, M., Martin, K., \&Suarez, H. (1995). Recurrent excitation in neocortical circuits. Science 269:981-985.

Douglas, R. J., \& Martin, K. A. C. (1990). Control of neuronal output by inhibition at the axon initial segment. Neural Comp. 2:283-292.

Douglas, R. J., Martin, K. A. C., \&Whitteridge, D. (1991). An intracellular analysis of the visual responses of neurones in cat visual cortex. J. Physiol.440:659-696.

Fuortes, M. G. F. (1959). Initiation of impulses in visual cells of Limulus. J. Physiol. 148:14-28.

Granit, R., Kernell, D., \& Lamarre, Y. (1966). Algebraical summation in synaptic activation of motoneurons firing within the "primary range" to injected currents. J. Physiol. 187:379-399.

Heeger, D. J. (1992). Normalization of cell responses in cat striate cortex. Vis. Neurosci. 9:181-197.

Heeger, D. J., Simoncelli, E. P., \& Movshon, J. A. (1996). Computational models of cortical visual processing. Proc. Natl. Acad. Sci. USA 93:623-627.

Hines, M. (1989). A program for simulation of nerve equations with branching geometries. Int. J. Biomed. Comp. 24:55-68.

Hines, M. (1993). The NEURON simulation program. In J. Skrzypek (Ed.), Neural Network Simulation Environments. Boston: Kluwer.

Hopfield, J. J. (1984). Neurons with graded response have collective computational properties like those of two-state neurons. Proc. Natl. Acad. Sci. USA 81:3088-3092.

Kernell, D. (1969). Synaptic conductance changes and the repetitive impulse discharge of spinal motoneurons. Brain Res. 15:291-294.

Koch, C., Bernander, Ö., \& Douglas, R. J. (1995). Do neurons have a voltage or a current threshold for action potential initiation? J. Comp. Neurosci. 2:63-82.

Koch, C., Poggio, T., \& Torre, V. (1982). Retinal ganglion cells: A functional interpretation of dendritic morphology. Proc. R. Soc. Lond. B 298:227-264.

Koch, C., Poggio, T., \& Torre, V. (1983). Nonlinear interaction in a dendritic tree: Localization timing and role in information processing. Proc. Natl. Acad. Sci. USA 80:2799-2802.

Nelson, M. E. (1994). A mechanism for neuronal gain control by descending pathways. Neural Comp. 6:242-254.

Payne, J. R., \& Nelson, M. E. (1996). Neuronal gain control by regulation of membrane conductance in two classes of spiking neuron models. Fifth Annual Computational Neuroscience Meeting Abstracts, p. 147.

Rapp, M., Yarom, Y., \& Segev, I. (1992). The impact of parallel fiber background activity on the cable properties of cerebellar Purkinje cells. Neural Comp. 4:518-533.

Rose, D. (1977). On the arithmetical operation performed by inhibitory synapses onto the neuronal soma. Exp. Brain Res. 28:221-223. 
Stein, R. B. (1967). The frequency of nerve action potentials generated by applied currents. Proc. R. Soc. Lond. B 167:64-86.

Torre, V., \& Poggio, T. (1978). A synaptic mechanism possibly underlying directional selectivity to motion. Proc. R. Soc. Lond. B 202:409-416.

Received August 19, 1996; accepted October 31, 1996. 